\title{
Diagnóstico y prevalencia de trastornos de la personalidad en atención ambulatoria. Estudio descriptivo.
}

The diagnosis and prevalence of personality disorders in outpatient services. A descriptive study.

\author{
Francesc Giner Zaragoza a , Guillem Lera Calatayud ' ${ }^{\text {, }}$ María Luisa Vidal Sánchez c, Mari Paz Pu- \\ chades Muñoz ${ }^{\mathrm{d}}$, Ainhoa Rodenes Pérez ${ }^{\mathrm{e}}$, Sonia Císcar Pons ${ }^{\mathrm{f}}$, Carlos Chiclana Actis ${ }^{\mathrm{g}}$, María Martín

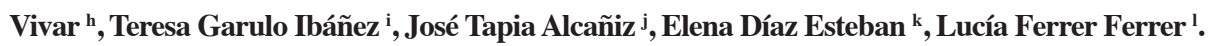 \\ ${ }^{a, b, d y \text { }}$ Psiquiatra. Hospital de La Ribera-Alzira. Valencia, España. ${ }^{e, i, j y l}$ Enfermera especializada en Psi- \\ quiatría. Hospital de La Ribera-Alzira. Valencia, España ${ }^{\text {eyf }}$ Psicóloga clínica. Hospital de La Ribera-Alzi- \\ ra. Valencia, España ${ }^{g}$ Psiquiatra. Consulta Dr. Carlos Chiclana. Madrid, España. ${ }^{h}$ Facultad de Medicina. \\ Universidad San Pablo CEU. Madrid, España.
}

Correspondencia: Guillem Lera Calatayud (guillemlera@gmail.com)

Recibido: 09/09/2014; aceptado con modificaciones: 21/04/2015

\begin{abstract}
RESUMEN: Introducción: los trastornos de personalidad (TP) son difíciles de diagnosticar. La literatura científica informa de su elevada prevalencia en muestras clínicas, pero en la práctica clínica diaria su diagnóstico es muy poco frecuente. Objetivos: Este estudio pretende estimar la prevalencia de TP en una Unidad ambulatoria de Salud Mental (USM), en situación real, para primeras citas. Método: Se aplicó el Cuestionario Salamanca (CS) como instrumento de cribado y, con los puntos de corte mínimo (2/3) y máximo (5/6), se comparó con el diagnóstico clínico realizado por un psicólogo clínico o psiquiatra, sin conocer el resultado del cribado con CS. Resultados: El diagnóstico de TP realizado por un profesional fue de un $7 \%$, frente al $100 \%$ de positivos obtenido por el CS en el punto de corte mínimo y el $53 \%$ con el máximo: 11,8\% para Clúster A, 19,8\% Clúster B y $21,4 \%$ en el C. Conclusiones: La elevada prevalencia de TPs en los centros de salud mental, junto con el infradiagnóstico clínico, son otros factores que ensombrecen el pronóstico de estos pacientes.

PALABRAS CLAVE: Trastornos de la personalidad, diagnóstico, cribado, prevalencia.
\end{abstract}

ABSTRACT: Introduction: Personality disorders (PD) are difficult to diagnose. Scientific literature shows a high prevalence in outpatient mental health services, but in daily practice the diagnosis of a PD is infrequent. Objectives: This study aims to calculate the prevalence of PD in outpatient mental health services in real clinic situation among patients who come for the first time. Method: Salamanca Questionnaire (SQ) was used as a screening method with the minimum score cut $(2 / 3)$ and the maximum score cut $(5 / 6)$. The results were compared with the clinical diagnose proposed by the psychiatrist or psychologyst, who did not know the result of SQ. Results: Only $7 \%$ of patients received PD diagnose although $100 \%$ of patients had pathological results with SQ using the lowest score proposed and 53\% using the highest score proposed: 11,8\% Clúster A, 19,8\%, Clúster B and 21,4\% Cluster C. Conclusions: The high prevalence of PD in outpatient mental health services and the low diagnosis rate are elements that worsen the prognosis of these patients.

KEY WORDS: Personality disorders, diagnosis, prevalence, mass screening.

\section{Introducción}

La evaluación de los trastornos de la personalidad (TP) en situación asistencial es una tarea compleja. Están infra-diagnosticados en la práctica clínica, los diagnósticos se solapan entre sí y están conceptualizados de tal manera que ningún modelo actual prevalece sobre otro (1-3).

En población general, con estudios realizados entre 1997-2007 y con muestras mayores de 250 personas, se estima la prevalencia entre el $4,4 \%$ y el $19 \%$ (4). Como 
dice Torgersen (5), los trastornos de la personalidad son prevalentes: más de 1 de cada 10 adultos padece un trastorno de la personalidad. Cuando dichas estimaciones se realizan en población psiquiátrica, la prevalencia aumenta considerablemente y se sitúa entre el $10,8 \%$ y el $82 \%$ (4). Los resultados de estudios realizados sobre población clínica española sitúan la prevalencia entre 3,7\% y 91\% (6).

Tabla 1.

Estimaciones de prevalencia de TP en muestras clínicas españolas.

\begin{tabular}{lllll}
\hline Autor(es) & Año & Población & Instrumento & Prevalencia \\
\hline Pedrero et al. & 2003 & Adictos Drogas & MCMI-II & $83 \%$ \\
Fernández-Montalvo et al. & 2004 & Adictos Drogas & MCMI-II & $76,20 \%$ \\
López et al & 2007 & Cárcel & IPDE & $69,30 \%$ \\
Del Rio et al & 2002 & Bulimia & MCMI-II & $81 \%$ \\
Fernández-Montalvo et al. & 2003 & Alcohol & MCMI-II & $64,20 \%$ \\
Fernández-Montalvo et al. & 2003 & Cocaína & MCMI-II & $45,70 \%$ \\
Fernández-Montalvo et al. & 2009 & Cocaína & MCMI-II & $36,70 \%$ \\
Fernández-Montalvo et al. & 2001 & Alcohol & MCMI-II & $62,80 \%$ \\
San Narciso et al, & 2000 & Heroína & IPDE & $42,90 \%$ \\
Martín et al. & 2009 & TCA & MCMI-II & $91 \%$ \\
López Torrecillas et al. & 2004 & Drogas & MCMI-II & $71,40 \%$ \\
González Trijueque et al. & 2008 & Acoso laboral & MCMI-II & $36,00 \%$ \\
Monras et al & 2008 & Alcohol & TCI & $12,40 \%$ \\
Jáuregui et al & 2009 & TCA & MCMI-II & varias* \\
Salavera et al & 2009 & Sin hogar & MCMI-II & varias* \\
Marañón et al. & 2007 & Muestra Psiquiátrica (84) & IPDE & $54,80 \%$ \\
Vázquez Roel y Cittadini & 2007 & Muestra Psiquiátrica (526) & DSM-IV/SCID-II & $40,10 \%$ \\
Baca-García et al. & 2007 & Muestra Psiquiátrica (10025) & CIE 10 & $3,70 \%$ \\
Chiclana et al & 2010 & Muestra Psiquiátrica (188) & IPDE & $39,40 \%$ \\
\hline Según estimacon & & \\
\hline
\end{tabular}

* Según estimación de cada prevalencia individual de TP.

Existen autores que afirman que los TP son el grupo de trastornos mentales que más frecuentemente atienden los psiquiatras (7-8), aunque no los diagnosticamos en el trabajo diario.

La infravaloración del diagnóstico de TP es máxima en Atención Primaria. Provoca que el porcentaje de pacientes diagnosticados de Trastorno Límite de la Personalidad (TLP) sea menor que el porcentaje estimado para la población general (9). Este caso es especialmente relevante, ya que el TLP es uno de los trastornos que más problemas de frecuencia elevada de contactos y de conflictividad presentan en el Sistema Nacional de Salud, actualmente saturado. Además, existe relación entre la presencia de TP y la evolución y abordaje de patologías (10-14) psiquiátricas y enfermedades médicas generales, además de influir en la hiperfrecuentación médica. Como ejemplos concretos y utilizando el Cuestionario Salamanca, se ha observado 
relación entre la migraña crónica y el TP anancástico, o entre la evolución de la placa aterosclerótica coronaria y los TP histriónico y ansioso. Con cuestionarios dimensionales, un patrón de introversión en pacientes en hemodiálisis periódica, entre otros.

Pero el diagnóstico de los patrones TP en situación asistencial es complicado $\mathrm{y}$, en general, solo resultan factibles los cuestionarios de cribado, contra las entrevistas clínicas semiestructuradas. Importa el tiempo y la utilidad clínica. El modelo más tradicional de cribado, denominado hallazgo de casos o cribado para la detección del trastorno, consiste en valorar a individuos aparentemente sanos, en este caso como TP, para identificar a aquellos con síntomas iniciales o avanzados del trastorno a evidenciar. Se utilizan cuestionarios que permiten focalizar la atención del profesional en determinados pacientes. Su utilidad reside en la capacidad para reducir al máximo los falsos negativos, con el riesgo de aumentar excesivamente los falsos positivos (15).

El diagnóstico clínico de los TP presenta abundantes dificultades.

Tabla 2:

Principales dificultades en la evaluación de los TP.

En el paciente

. Resistencia del paciente a ser calificado como TP.

En el clínico

. Falta de formación específica sobre los TP

- Basarse en el diagnóstico previo realizado por otro clínico sin ser crítico

. Confundir psicopatología del Eje I con la del Eje II

- Obtener la información de manera inadecuada

- Basar el diagnóstico en la contratransferencia negativa que el paciente provoca

- Aplicar las categorías DSM o CIE mecánicamente sin un análisis suficientemente profundo

- No aceptar la existencia de los TP como entidad clínica independiente

. Considerar los TP como si fueran "desviaciones morales"

\section{En el TP}

Falta de un consenso sobre el concepto mismo de la enfermedad

. Presencia de un trastorno del Eje I o de una enfermedad médica

\section{En el método de evaluación}

. Dificultades para distinguir entre variaciones de la personalidad normal y TP

. Ausencia de una prueba diagnóstica universalmente aceptada

Entre ellos, los instrumentos de screening no advierten numerosas fuentes de distorsión, como la simulación, la exageración o el ocultamiento. En una unidad ambulatoria, los cuestionarios son útiles como complemento a los criterios clínicos.

Las entrevistas diagnósticas semi-estructuradas, validadas para el diagnóstico de TP, son muy difíciles de emplear en la práctica diaria, o imposible por el tiempo que exige en un ambiente de consulta ambulatoria. También se ven afectadas por factores de distorsión $(16,17)$. 
Cuando hablamos de TP, esta distorsión puede ser mayor debido al carácter egosintónico de algunos de los síntomas. Para solucionar este problema, no es eficaz aumentar el número de cribadores (18), pero según un estudio realizado por Korzekwa (19) para el diagnóstico de TLP, sí es eficaz el emplear una metodología en 2 pasos, primero un cribado con test autoaplicado y después administrar la entrevista DIB-R. Los resultados de este estudio fueron un diagnóstico del $72 \%$ en el screening y $22 \%$ en el segundo paso.

Nosotros observamos otros factores que obstaculizan el diagnóstico en la práctica cotidiana asistencial.

Tabla 3:

Principales factores detectados en la dificultad del diagnóstico de TP por el equipo investigador, 2014.

. Presión asistencial.

. Frecuente implicación médico-legal con pacientes problemáticos.

. Tiempo necesario para informar a terceros sobre las capacidades y responsabilidades de los pacientes.

. Formación clásica de centrarse en enfermedades del Eje I.

. Carácter frecuentemente egosintónico de los rasgos.

. La inexistencia de tratamientos farmacológicos específicos puede "descorazonar" al clínico, que prefiere "no ver".

. Imposibilidad de ofrecer psicoterapias validadas, reglads, en las USM para TPs.

. Falta de tiempo.

. Escasa fiabilidad de los instrumentos de medida asequibles al profesional.

. Complejidad conceptual, que ahuyenta al clínico de realizar diagnósticos que dificulten su día a día.

En España disponemos de 2 cuestionarios de cribado categoriales: el Cuestionario Salamanca (20) y las versiones reducidas del IPDE (21). Prieto Etxebeste y cols. (22), en una muestra de adicciones, observaron que la concordancia de resultados entre los dos instrumentos es muy alta, especialmente en pacientes que presentan altas puntuaciones: 4 o más ítems en IPDE-77 y resultados 5/6 en CS, con una mayor dispersión para resultados de corte más bajo (2/3 en ambos instrumentos).

La mayor parte de los estudios en España se han realizado en muestras clínicas con patologías específicas. No hemos encontrado ningún trabajo con una muestra que represente la práctica clínica habitual: diagnóstico psiquiátrico o psicológico en las primeras consultas de una Unidad ambulatoria de Salud Mental.

Nos planteamos estimar la prevalencia de TP, con el CS, en una muestra de población clínica ambulatoria y describir su distribución por cluster, de acuerdo con las variables sociodemográficas y médico-psiquiátricas recogidas.

\section{Material y Método}

La inclusión de participantes en el estudio se realiza en un medio asistencial público ambulatorio (USM de Sueca. Departamento de Salud de La Ribera) que pro- 
porciona asistencia psiquiátrica y psicológica ambulatorias a una población de aproximadamente 100.000 habitantes.

A los pacientes que pueden ser incluidos y no cumplen los criterios de exclusión (Tabla 4), se les propone participar, sin coste adicional para ellos y sin recibir remuneración económica alguna por dicha participación.

Tabla 4.

Criterios de exclusión.

1. Dificultad para comprender los enunciados del cuestionario por disfunción cognitiva o problemas con el idioma.

2. Encontrarse afectado, durante la participación en el estudio, por un episodio psicótico o algún otro estado que pudiera alterar su juicio de realidad (según valoración del DUE especialista en Psiquiatría).

3. Reticencia a participar (si existiesen dudas en el paciente, a pesar del cumplimiento de los criterios de inclusión, no se insistiría en su participación).

4. Ser menor de 18 años en el momento de su participación en el estudio.

5. Riesgo de auto o hetero agresión (según valoración del personal clínico).

6. Presentar una patología orgánica que diera lugar a manifestaciones sintomáticas susceptibles de ser confundidas con rasgos patológicos de la personalidad.

Siguen un muestreo de conveniencia: las primeras consultas durante los 6 primeros meses de 2012 con los psiquiatras y psicólogos de la Unidad. Se van incluyendo de manera progresiva, consecutivamente, según llegan a la primera entrevista, aceptan o no participar y firman el consentimiento informado tras una explicación completa de la metodología y objetivos del estudio.

Se recoge la prueba completada, antes de ser entrevistados por el psiquiatra o psicólogo, quien determina su impresión diagnóstica sin conocer el resultado del cribado. De esta forma, se evita el sesgo que puede provocar sobre-interpretación cuando la prueba de cribado es positiva y su infra-interpretación cuando es negativa (23).

El CS es un cuestionario autoaplicado formado por 22 ítems, con un tiempo de realización entre 5 y 10 minutos. Cada ítem presenta una respuesta dicotómica, verdadero o falso. Esta prueba ofrece la clásica agrupación diagnóstica en 3 grandes clústeres: A o "excéntrico/psicótico", B o "dramático/borderline" y C o "ansioso/ neurótico". La prueba permite diferenciar 11 categorías de TP. Dentro de estas categorías, se incluyen las diferencias de criterio entre CIE 10 y DSM-IV-TR: El TLP se denomina Trastorno de Inestabilidad Emocional (TIE) y lo diferencia en 2 categorías: TIE subtipo impulsivo y TIE subtipo límite. Al TP Obsesivo lo denomina Trastorno Anancástico y al Trastorno Evitativo, Trastorno Ansioso. No es un cuestionario validado en población general en el momento de escribir este artículo. Por su fácil uso en la práctica clínica, ha sido usado en las aportaciones de investigación españolas sobre prevalencia de TP en muestras clínicas. Los autores establecen el punto de corte para cada TP en $2 / 3$.

En una Unidad asistencial de Salud Mental, el tiempo es escaso, por lo que el cribado debe ser mínimamente útil, es decir, debe discriminar al menos a los pacien- 
tes más afectados. Por ello, decidimos aumentar el punto de corte al máximo permitido por el CS, de 5/6, para reducir en la medida de lo posible los falsos positivos. Se compararon los resultados con el punto de corte propuesto por los autores del instrumento, de $2 / 3$.

De la historia clínica electrónica, se recogieron distintas variables sociodemográficas: edad, sexo, número de contactos con los servicios de urgencias, con quién reside. El diagnóstico principal, motivo de demanda asistencial, se agrupó en 9 grandes categorías para facilitar su visibilidad.

Se empleó el programa SPSS 19.0 para Windows (SPSS Inc., Chicago, III, USA) para el cálculo de estadísticos descriptivos.

\section{Resultados}

Doscientas veinte personas aceptan participar. Tras excluir a los que no habían completado adecuadamente el cuestionario, la muestra final está compuesta por 187 sujetos (64,2\% mujeres), con una media de edad de 44 años. La mayoría $(85,5 \%)$ reside en el entorno familiar, el 9,6\% sólo y el 4,5\% de otras maneras.

Los diagnósticos principales más frecuentes son trastornos ansiosos (31\%) y trastornos adaptativos (28,9\%). La media de contactos con urgencias es de 17 .

Sólo el 7\% de los pacientes son diagnosticados como TP, como diagnóstico principal, después de la entrevista realizada con el psiquiatra o psicólogo, sin que estos supieran los resultados del CS para el paciente que entrevistaban.

Tabla 5 .

Diagnóstico principal realizado por el profesional clínico tras entrevista.

\begin{tabular}{|lc}
\hline Trastornos ansiedad, somatomorfos, facticios, disociativos, sexuales y del sueño & $31,0 \%$ \\
Trastornos adaptativos & $28,9 \%$ \\
Trastornos del estado del ánimo & $19,3 \%$ \\
Trastornos de la personalidad & $7,0 \%$ \\
Otros códigos, incl. trast. inicio infancia/adol. & $5,8 \%$ \\
Esquizofrenia y otra psicosis & $2,7 \%$ \\
Trastorno por el uso de sustancias y otras adicciones & $2,1 \%$ \\
Trastornos de la conducta alimentaria & $1,1 \%$ \\
Deterioro cognitivo leve/otros relacionados & $2,1 \%$ \\
\hline
\end{tabular}

Tras la aplicación del CS y teniendo en cuenta el punto de corte $2 / 3$ recomendado, el $100 \%$ de la muestra presenta al menos 1 TP. Los más prevalentes son el Histriónico (65,8\%), Ansioso-Evitativo (62,6\%) y Esquizoide (41,2\%). 
Aplicando el punto de corte máximo (5/6): el 53\% de la muestra presenta al menos un TP. Aquí, los más frecuentes son el Ansioso (12,8\%), el TIE-Límite (8,0\%), el Esquizoide (8,0\%), el Dependiente (7,5\%) y el TIE-Impulsivo (7\%) (Tabla 6).

El TIE, incluyendo los dos subtipos Impulsivo y Límite, es el más prevalente en nuestra muestra, empleando el punto de corte máximo, con un valor del 15\%.

Tabla 6.

Prevalencia de TP (\%) en clúster y TP especificos, según puntos de corte CS.

\begin{tabular}{lll}
\hline & Mínimo (2/3) & Máximo (5/6) \\
\hline Clúster A & \\
\hline Esquizoide & $41,2 \%$ & $8,0 \%$ \\
Paranoide & $23,0 \%$ & $2,1 \%$ \\
Esquizotípico & $18,2 \%$ & $3,2 \%$ \\
Total n (\%) & $103(55.1 \%)$ & $22(11,8 \%)$ \\
\hline Clúster B & & \\
\hline Histriónico & $65,8 \%$ & $7,5 \%$ \\
TIE-I & $43,9 \%$ & $7,0 \%$ \\
TIE-L & $43,3 \%$ & $8,0 \%$ \\
TIE-I + TIE-L & $87,2 \%$ & $15 \%$ \\
Narcisista & $13,4 \%$ & $2,1 \%$ \\
Antisocial & $3,7 \%$ & $0,0 \%$ \\
Total B n (\%) & $187(100 \%)$ & $37(19,8 \%)$ \\
\hline Clúster C & & \\
\hline Ansioso & $62,6 \%$ & $12,8 \%$ \\
Dependiente & $47,6 \%$ & $7,5 \%$ \\
Anancástico & $39,6 \%$ & $6,4 \%$ \\
Total n (\%) & $145(77,5 \%)$ & $40(21,4 \%)$ \\
\hline Al menos 1 TP n (\%) & $187(100 \%)$ & $99(53 \%)$ \\
\hline
\end{tabular}

También, aplicamos el punto de corte máximo (5/6) para las variables médicopsiquiátricas, según clusters.

Tabla 7.

Frecuencia (\%) de contactos en urgencias y de diagnósticos en eje I agrupados, por clusters y según punto de corte máximo del CS, 5/6.

\begin{tabular}{llll}
\hline & $\begin{array}{l}\text { Cluster A } \\
(\mathrm{n}=22)\end{array}$ & $\begin{array}{l}\text { Cluster B } \\
(\mathrm{n}=37)\end{array}$ & $\begin{array}{l}\text { Cluster C } \\
(\mathrm{n}=40)\end{array}$ \\
\hline $\begin{array}{l}\text { Media contactos } \\
\text { urgencias }\end{array}$ & 16,95 & 20,92 & 20,18 \\
T ansiedad/otros & $18,2 \%$ & $35,1 \%$ & $30,0 \%$ \\
Tr. adaptativos & $18,2 \%$ & $18,9 \%$ & $22,5 \%$ \\
Tr. ánimo & $31,8 \%$ & $18,9 \%$ & $25,0 \%$ \\
Esquizofrenia/otr & $4,5 \%$ & $5,4 \%$ & $2,5 \%$ \\
\hline Tr. personalidad & $4,5 \%$ & $5,4 \%$ & $2,5 \%$ \\
\hline Adicciones & $4,5 \%$ & $5,4 \%$ & $5,0 \%$ \\
Tr. c. alimentaria & $4,5 \%$ & $5,4 \%$ & $2,5 \%$ \\
Otros códigos $/ \mathrm{Tr}$ & $13,8 \%$ & $2,7 \%$ & $7,5 \%$ \\
infancia/ adolesc. & & & \\
Det. cognitivo & $0,0 \%$ & $2,7 \%$ & $2,5 \%$
\end{tabular}


ORIGINALES Y REVISIONES

\section{Discusión}

El CS, en nuestra muestra de pacientes ambulatorios, presenta una elevada sensibilidad, obteniendo un exceso de falsos positivos, no resultando fiable para las personas que informan de rasgos relacionados con la presencia de TP $(100 \%$ de la muestra para $1 \mathrm{TP}$ al menos). Parece que el punto de corte, 2/3, propuesta por los autores del CS, es muy bajo para que sea útil en la práctica clínica cotidiana de una USM (CSM).

La prevalencia de TP con el CS, en nuestra muestra y empleando el punto de corte máximo de $5 / 6$, es de nuevo muy elevada (53\%). Sin embargo, estos pacientes no se diagnostican ni se tratan como tal y sólo el 7\% de diagnósticos clínicos principales fue de TP.

La alta prevalencia con ambos puntos de corte puede ser debida al llamado sesgo de estado, es decir, cuando los instrumentos de cribado de los TP distorsionan los resultados al reflejar estados propios de una patología del Eje I. Así, en la patología aguda en primer plano, sin tratar (son pacientes que acuden por primera vez o reanudan contacto en la Unidad), el diagnóstico por cuestionario discrimina mal las anomalías transitorias o variaciones de estado (discriminación estado-rasgo) (24).

En especial, los ítems que informan de rasgos correspondientes al grupo de personalidades ansiosas y dramáticas, pueden ser entendidos por el paciente que acude por primera vez a una USM, en un momento de crisis, por estados patológicos actuales, agudos, como malestar emocional motivo de la demanda de asistencia.

Esto parece hacerse evidente, en nuestra muestra, para los sujetos con rasgos autoinformados del cluster B, en concreto para el 15\% de pacientes TIE (subtipo Impulsivo + subtipo Límite), los más prevalentes usando el punto de corte máximo del cuestionario. Este hallazgo puede resultar de utilidad para analizar la valoración que hace el paciente inestable emocionalmente de qué le ocurre y de cómo formula su problema en su primera visita en Salud Mental. Prima el síntoma egodistónico sobre el rasgo egosintónico. Pero esta distorsión, donde el estado oculta el rasgo, confunde la información relevante para el diagnóstico del TIE, o del TP Histriónico o Ansioso. Es necesaria una observación longitudinal y una recogida de información también de la familia o del entorno próximo. Los TP son "pacientes difíciles" con los que, de forma inconsciente, podemos evitar "ver" su diagnóstico en Eje II para centrarnos en lo inmediato: sintomatología a categorizar en Eje I. La espera necesaria para la observación clínica longitudinal con la que diagnosticar adecuadamente un patrón de TP que se mantiene durante años puede favorecer la negligencia no voluntaria del equipo asistencial, los familiares y los propios pacientes.

Un dato de especial relevancia en nuestra muestra es que las personalidades "dramáticas o emocionales" del cluster B, hiperfrecuentan los servicios de urgencias, tanto en Atención Primaria como hospitalaria. La media de contactos en los servicios 
de urgencias es, respectivamente, para los grupos A, B y C, de 16,95, 20,92 y 20,18 desde el período de inicio de la Historia Clínica Electrónica hasta el de la realización del estudio (período 2000-2012). Muchos contactos, y ello que estamos empleando el punto de corte máximo que permite el CS.

Estas personas muestran déficits continuados y persistentes en sus capacidades de relación interpersonal (25), de tal manera que un elevado número de ellos presentan problemas de salud mental graves, patologías del Eje I, agresividad o ideación suicida. Es casi definitorio el malestar emocional intenso del TIE, generalmente tras conflictos con personas significativas de su entorno. Como dice Skodol (26), los pacientes con TLP probablemente experimentan y expresan un malestar personal considerable, especialmente cuando se sienten decepcionados por alguien significativo. La consulta urgente (voluntariamente, por presión familiar o tras acto parasuicida, entre otros) suele ser la consecuencia. Es difícil lograr y mantener un funcionamiento estable y adecuado en estos pacientes, especialmente complicado a medio y largo plazo en el aspecto ocupacional (27). Todo ello es causa de grave deterioro personal, familiar y social.

\section{Conclusiones}

Conociendo las limitaciones metodológicas de nuestro estudio, observamos que, en nuestra muestra, la prevalencia de TP con el CS, empleando el punto de corte máximo, es muy elevada (53\%). Sin embargo, estos pacientes no se diagnostican ni se tratan como tal y sólo el 7\% de diagnósticos clínicos principales fue de TP. Concuerda este hecho con lo aportado por otros autores (28). La falta de atención sobre los rasgos desadaptativos de los pacientes complica el tratamiento y tiene importantes implicaciones terapéuticas y de pronóstico. La peor evolución está probada tanto para sus enfermedades médicas o psiquiátricas, como para la evolución general, biopsicosocial, de la persona con TP. Los TIE están especialmente infradiagnosticados y presentan, de por sí, un elevado malestar emocional que repercute tanto en ellos como en su entorno. Para mejorar su asistencia psiquiátrica, es preciso contar con un cribado diagnóstico sensible y específico, que facilite al clínico la decisión de a quién seguir evolutivamente de forma más directa. Serían útiles análisis más sofisticados para establecer perfiles sociodemográficos y clínicos que discriminen más riesgo de presencia de TP. También son necesarios más estudios de prevalencia en medio clínico real, con muestras amplias, enfatizando la importancia de realizarlos en las unidades de primera línea, como el extra-hospitalario de la USM y de los Centros de Salud de Atención Primaria. 


\section{BIBLIOGRAFÍA}

(1) Bernardo Arroyo M, Roca Benassar, M. Trastornos de la Personalidad. Evaluación y Tratamiento. Barcelona: Masson SA; 1998.

(2) Mirapeix C. De la multicausalidad bio-psico-social a una concepción integradora de la psicoterapia. Archivos de Neurobiología. 1993; editorial.

(3) Vallejo Ruiloba J, Baca Baldomero E, Leal Cercós C. Modelos conceptuales en la psiquiatría actual. En: Vallejo Ruiloba J, Leal Cercós C, editores. Tratado de Psiquiatría. $2^{\text {a }}$ edición. Barcelona: Ars Medica; 2010. p. 63-78.

(4) Chiclana C, Rodríguez T, Aubá E. Trastornos de Personalidad. En: Ortuño F editor. Lecciones de Psiquiatría. $1^{\text {a }}$ ed. Madrid: Panamericana; 2010. p. 257-284.

(5) Torgersen S. Epidemiología. En: Oldham JM, Skodol AE, Bender DS, directores. Tratado de los Trastornos de la Personalidad. $1^{\text {a }}$ ed. Barcelona: Elsevier Masson; 2007. p. 131-143.

(6) Chiclana C. Trastornos de la Personalidad: expresión dimensional del diagnóstico categorial [Tesis Doctoral]. Pamplona: Departamento de Psiquiatría y Psicología Médica. Facultad de Medicina. Universidad de Navarra; 2010.

(7) Zimmerman M, Rothschild L, Chelmisky I. The prevalence of DSM-IV personality disorders in psychiatric outpatients. American Journal of Psychiatry, 2005, 162: 1911-1918.

(8) Zimmerman M, Chelminski I, Young D. The frequency of personality disorders in psychiatric patients. Psychiatr Clin North Am. 2008; 31(3): 405-420.

(9) Aragonés E, Salvador-Carulla L, López-Muntaner J. Prevalencia registrada del trastorno límite de personalidad en las bases de datos de Atención Primaria. Gac Sanit. 2013; 27(2): 171-174.

(10) Frankenburg FR, Zanarini MC. Personality disorders and medical comorbidity. Curr Opin Psychiatry. 2006; 19: 428-431.

(11) Rodríguez Muñoz C, Cebrià Andreu J, Corbella Santomá S, Segura Bernal J, Sobrequés Soriano J. Rasgos de personalidad y malestar psíquico asociados a los pacientes hiperfrecuentadores de consultas de Atención Primaria. MEDIFAM. 2003; 13: 143-150.

(12) Muñoz I, Toribio-Díaz E, Carod-Artal FJ, Peñas-Martínez ML, Ruiz L, Domínguez E, et al. Rasgos de personalidad en pacientes migrañosos: estudio multicéntrico utilizando el cuestionario de cribado Salamanca. Rev Neurol. 2013; 57: 529-534.

(13) Suarez-Bagnasco M, Ganum G, Cerda M. Trastornos de personalidad y placa aterosclerótica coronaria. Rev argent cardiol [Internet]. 2013 [citado 4 Oct 2014]; 81 (1): p. 39-44. Disponible en: http://www.scielo.org.ar/scielo.php?script=sci_abstract\&pid=S1850-37482013000100007\&lng=pt.

(14) Montejo González AL, Sánchez García JM, Ginés Llorca R. Estudio del estado de salud, comorbilidad depresiva y rasgos de personalidad en pacientes en hemodiálisis renal crónica. Psiq Biol. 2006; 13(2): p. 67-70.

(15) McDermut W, Zimmerman M. Instrumentos de evaluación y evaluación estandarizada. En: Oldham JM, Skodol AE, Bender DS, directores. Tratado de los Trastornos de la Personalidad. 1 $^{\text {a }}$ ed. Barcelona: Elsevier Masson; 2007. p. 91-103.

(16) Fernández-Montalvo, J, Echeburúa E. Uso y abuso de los autoinformes en la evaluación de los trastornos de personalidad. Revista de Psicopatología y Psicología Clínica. 2006;1 (11):1-12.

(17) Echeburúa E, Amor PJ, Corral P. Autoinformes y entrevistas en el ámbito de la psicología clínica forense: limitaciones y nuevas perspectivas. Análisis y Modificación de Conducta. 2003;29:503-522. 
(18) Morse JQ, Plikonis PA. Screening for Personality Disorders. J Pers Disor. 200;21(2):179198.

(19) Korzekwa MI, Dell PF, Links PS, Thabane L, Webb SP. Estimating the prevalence of borderline personality disorder in psychiatric outpatients using a two-phase procedure. Comprehensive Psychiatry. 2008;49: 380-386.

(20) García-Portilla MP, Bascarán MT, Sáiz PA, Parellada M, Bousoño M, Bobes J. Instrumentos de evaluación para la personalidad y sus trastornos. Cuestionario Salamanca de Trastornos de la Personalidad, Pérez-Urdániz A, Rubio V, Gómez ME autores. En: García-Portilla MP, Bascarán MT, Sáiz PA, Parellada M, Bousoño M, Bobes J, directores. Banco de instrumentos básicos para la práctica de la psiquiatría clínica. $6^{\text {a }}$ ed. Majadahonda: Comunicación y Ediciones sanitarias SL; 2011.p. 204-223.

(21) García-Portilla MP, Bascarán MT, Sáiz PA, Parellada M, Bousoño M, Bobes J. Instrumentos de evaluación para la personalidad y sus trastornos. IPDE. International Personality Disorder Examination, version Española, López-Ibor J, Pérez-Urdániz A, Rubio V, autores. En: García-Portilla MP, Bascarán MT, Sáiz PA, Parellada M, Bousoño M, Bobes J, directores. Banco de instrumentos básicos para la práctica de la psiquiatría clínica. $6^{\mathrm{a}}$ ed. Majadahonda: Comunicación y Ediciones sanitarias SL; 2011.p. 204-223.

(22) Prieto Etxebeste M, Sanz Etxeberria J, Landabaso Vazquez M, Garnica de Cos E, Sánchez Pérez M. Cuestionario de Personalidad IPDE y Cuestionario Salamanca. Estudio comparativo en una muestra de pacientes con trastorno por uso de sustancias. [Internet] [Poster]. X Congreso Nacional Psiquiatría. Sevilla. 2006. Disponible en: http://www.sepsiq.org/psiquiatriasevilla06/programa_indice.html.

(23) Strauss SE, Richardson WS, Glasziou P, Haynes RB. Medicina Basada en Evidencias. Como practicar y enseñar la MBE. 3a ed. Barcelona: Elsevier España; 2006.

(24) Cañas de Paz. Instrumentos de evaluación. En: Bernardo Arroyo M, Roca Benassar M. Trastornos de la Personalidad. Evaluación y Tratamiento. Barcelona: Masson; 1998. p. 77-96.

(25) Horowitz LM, Wilson KR, Turan B, Zolotsev P, Constantino MJ, Henderson L. How Interpersonal Problems Motives Clarify the meaning of Interpersonal Behaviour: a revised circumplex model. Model Personality and Social Psychology Review. 2006;1: 67-86.

(26) Skodol E. Manifestaciones, diagnóstico clínico y comorbilidad. En: Oldham JM, Skodol AE, Bender DS. Tratado de los Trastornos de la Personalidad. 1 ${ }^{a}$ ed. Barcelona: Elsevier Masson; 2007. p. 59-90.

(27) Zanarini MC, Frankenburg FR. The 10-year course of psychosocial functioning among patients with borderline personality disorder and axis II comparison subjects. Acta Psychiatr Scand. 2010;122(2):103-109.

(28) Chiclana Actis C, Lahortiga Ramos F. Trastornos de la Personalidad: Tratar o hacer la vista gorda. Psiquiatria.com [Internet]. 2011 [citado 04Oct 2014]; 15:50. Disponible en: http://hdl. handle.net/10401/4746. 